\title{
Minimizing donor site morbidity using the interfascicular nerve splitting technique in single-stage latissimus neuromuscular transfer for facial reanimation: a retrospective study
}

\author{
Seong Oh Park ${ }^{1}$, Jaewoo Kim², Il-Kug Kim ${ }^{3}$, Jee Hyeok Chung ${ }^{2}$, Ung Sik Jin², and Hak \\ Chang $^{2}$ \\ ${ }^{1}$ Hanyang University College of Medicine \\ ${ }^{2}$ Seoul National University College of Medicine \\ ${ }^{3}$ Yeungnam University Medical Center
}

June 17, 2020

\begin{abstract}
Introduction Free muscle transfer for facial reanimation requires the sacrifice of motor nerves and muscles, which inevitably leads to donor site morbidity. To overcome this, the authors performed the interfascicular nerve splitting technique during neurovascular latissimus dorsi flap harvest. The aim of this study was to examine the efficacy of our interfascicular nerve splitting technique through the evaluation of donor site morbidity. Methods Records of patients in who the free latissimus dorsi flap was employed with the interfascicular nerve splitting technique between 2012 and 2016 were reviewed. Postoperative donor site morbidity was evaluated using electromyography, nerve conduction studies, and the Quick-Disabilities of Arm, Shoulder, and Hand questionnaire (QuickDASH). Results A total of 13 patients were analyzed. Grades from the electromyography and nerve conduction study were not significantly different between the donor site and contralateral side $(0.42 \pm 0.51$ and $0.08 \pm 0.28$, respectively, $\mathrm{P}=.073$ ). QuickDASH scores showed different results over time. Preoperative QuickDASH scores averaged 1.57 \pm 2.34 . At postoperative 6 months, the average QuickDASH score was $8.74 \pm 4.62$, which was significantly different from the preoperative average $(\mathrm{P}=.001)$. At postoperative 12 months, QuickDASH scores averaged 2.62 \pm 3.19 , which was an improvement from the postoperative 6-month score. However, the improvement was not significantly different from the preoperative score $(\mathrm{P}=.059)$. Conclusion The present study showed that interfascicular nerve splitting could minimize donor site morbidity. Moreover, our results suggest that the split nerve can function as a donor nerve. Our novel method could be a valuable option for minimizing donor site morbidity during facial reanimation surgery.
\end{abstract}

Minimizing donor site morbidity using the interfascicular nerve splitting technique in singlestage latissimus neuromuscular transfer for facial reanimation: a retrospective study

\section{ABSTRACT}

Introduction

Free muscle transfer for facial reanimation requires the sacrifice of motor nerves and muscles, which inevitably leads to donor site morbidity. To overcome this, the authors performed the interfascicular nerve splitting technique during neurovascular latissimus dorsi flap harvest. The aim of this study was to examine the efficacy of our interfascicular nerve splitting technique through the evaluation of donor site morbidity.

\section{Methods}

Records of patients in who the free latissimus dorsi flap was employed with the interfascicular nerve splitting technique between 2012 and 2016 were reviewed. Postoperative donor site morbidity was evaluated 
using electromyography, nerve conduction studies, and the Quick-Disabilities of Arm, Shoulder, and Hand questionnaire (QuickDASH).

\section{Results}

A total of 13 patients were analyzed. Grades from the electromyography and nerve conduction study were not significantly different between the donor site and contralateral side $(0.42 \pm 0.51$ and $0.08 \pm 0.28$, respectively, $P=.073)$. QuickDASH scores showed different results over time. Preoperative QuickDASH scores averaged $1.57 \pm 2.34$. At postoperative 6 months, the average QuickDASH score was $8.74 \pm 4.62$, which was significantly different from the preoperative average $(P=.001)$. At postoperative 12 months, QuickDASH scores averaged $2.62 \pm 3.19$, which was an improvement from the postoperative 6 -month score. However, the improvement was not significantly different from the preoperative score $(P=.059)$.

\section{Conclusion}

The present study showed that interfascicular nerve splitting could minimize donor site morbidity. Moreover, our results suggest that the split nerve can function as a donor nerve. Our novel method could be a valuable option for minimizing donor site morbidity during facial reanimation surgery.

Keywords: Facial reanimation; Dual-innveration; Nerve splitting; Donor site morbidity

\section{Key Points:}

- This study retrospectively examined the efficacy of interfascicular nerve splitting technique through the evaluation of donor site morbidity.

- Interfascicular nerve splitting could minimize donor site morbidity.

- The split interfascicular nerve can function as a donor nerve.

- The distal stump of the thoracodorsal nerve has made dual innervation possible without any sacrifice of further site morbidity.

- Our novel method could be a valuable surgical option for facial reanimation to minimize donor site morbidity.

\section{Introduction}

In long-standing facial palsy, the latissimus dorsi and gracilis neuromuscular flaps are the most commonly used flaps for facial reanimation. Unlike the gracilis, the latissimus dorsi has a long neurovascular pedicle that can be used in various ways. ${ }^{1}$ However, unlike a perforator flap, free muscle transfer for facial reanimation requires the sacrifice of motor nerves and muscles, which inevitably leads to donor site morbidity. Latissimus dorsi harvest often induces donor site functional morbidity in the form of shoulder weakness and limitations in range of motion, daily living and sports activities. ${ }^{2}$ Only a small portion of muscle is needed for facial reanimation but motor nerves that innervate the latissimus dorsi muscle need to be sacrificed, especially for the dual-innervation technique, which is a common procedure. ${ }^{1,3}$ In spite of a recent study that showed minimal donor site functional morbidity, some patients reported discomfort, especially during sports or art activities. $^{4}$

For overcoming donor site morbidity, we performed an interfascicular nerve splitting technique during neurovascular latissimus dorsi muscle flap harvest. The possibility of this technique was first confirmed with an anatomic study. ${ }^{5}$ With this method, the descending and transverse branch of the thoracodorsal nerve (TDN) can be split without injury to the perineurium A long nerve with a length similar to that of the vascular pedicle can be obtained without unnecessary sacrifice of nerves. Interfascicular nerve splitting allows innervation of the remaining muscles, and at the same time, enables harvest of sufficient nerve length. The aim of this study was to examine the efficacy of our interfascicular nerve splitting technique through the evaluation of donor site morbidity.

\section{Patients and Methods}

This investigation was approved by the Institutional Review Board [removed for blind peer review]. This 
study was performed in accordance with the Declaration of Helsinki. We examined all patients that underwent free latissimus dorsi flap harvest for facial reanimation using the interfascicular nerve splitting technique between January 2012 and December 2016. All patients were diagnosed with irreversible, complete facial palsy and an estimated House-Brackmann grade 6 (total paralysis). Patients with insufficient medical records or whose follow-up period was less than 24 months were excluded. Patients who underwent procedures that could affect lip elevation (face-lift or sling procedure) were excluded. Patients who underwent only periorbital procedures (brow-lift or canthopexy) were included. Data were collected based on a retrospective chart review and included age at operation, etiology of the palsy, duration of paralysis, follow-up period, postoperative results, and donor site outcome and morbidity (Table 1).

\section{Surgical Technique}

A schematic illustration of the key procedure is shown in Figure 1. The patient was placed in a semilateral position with the paralyzed side up. A modified Blair incision was made on the paralyzed side and the cheek skin was elevated from the parotid fascia and superficial muscular aponeurotic system. The facial artery and vein were dissected and exposed. The masseteric motor nerve was exposed according to conventional methods. ${ }^{6} \mathrm{We}$ confirmed the twitch of masseter muscle using a nerve stimulator. On the contralateral side, an incision was made along the nasolabial fold to find the buccal branch. We also confirmed the excursion of the mouth corner. The length between the zygomatic arch and mouth corner of the paralyzed side was measured to determine the amount of muscle to harvest.

Incision was made on a posterior axillary line and an anterior border of the latissimus dorsi was confirmed. Thoracodorsal vessels and TDN were identified. Additional dissection was performed until reaching the bifurcation of thoracodorsal vessels and nerve near the hilum. We could identify the bifurcation of the descending branch and transverse branch of thoracodorsal vessels and TDN. After marking the required size on the muscle, additional dissection above the distal end of the muscle was performed in order to find the distal stump of the descending branch of the TDN. Approximately $5 \mathrm{~cm}$ of the distal stump was harvested with muscle. After separating the muscle, interfascicular nerve splitting of the TDN was performed to the maximum extent possible under microscopy. We confirmed the twitch of the muscle by stimulation of a split motor nerve using a nerve stimulator before dividing the pedicle (Figure 2) [See Video, Supplementary Content 1]

Microvascular anastomosis was performed. The proximal TDN was coapted with the buccal branch of the contralateral side by end-to-end epineural suture. The distal stump of the descending branch of the TDN was coapted with the ipsilateral masseteric motor nerve by end-to-end epineural suture. The proximal end of muscle was sutured to the modiolus and the distal end of muscle was fixed to the zygomatic arch with suturing to the periosteum.

\section{Outcome measures}

Postoperative results of facial reanimation were assessed approximately 24 months post-surgery using evaluation criteria from the study by Harii et al. ${ }^{1}$ [See Table, Supplementary Content 2]. For all patients, the preoperative grade was set at 1 (no correction). Quantitative measurement was performed using Hadlock's Smile Measurement of Improvement in Lip Excursion scale. ${ }^{7}$ For this, we utilized Emotrics, a python-based application, from the Sir Charles Bell Society ${ }^{8}$ [See Figure, Supplementary Content 3]

The donor site outcome and morbidity were measured by two aspects: (1) objective findings using electromyography (EMG) and nerve conduction study (NCS) and (2) subjective assessment using the Quick-Disabilities of Arm, Shoulder, and Hand (QuickDASH) questionnaire.

\section{Electromyography and Nerve Conduction Study}

To measure the latissimus dorsi muscle activity and the remaining TDN condition objectively at 12 months postoperatively, needle EMG and NCS were performed. For EMG, patients were put in a prone position, arm on the side and palm face up. Electrodes were inserted three fingerbreadths distal to and along the posterior axillary fold. Patients were asked to rotate internally, adduct, and extend the arm. For NCS, a 
standard concentric needle electrode was inserted into the belly of the latissimus dorsi at the posterior wall of the axilla. Stimulation was performed at Erb's point with a surface stimulator. Electrophysiologic changes related to the severity of lesions were scored into 6 grades according to EMG and NCS findings (Table 2). We compared donor site with contralateral side according to these scores.

\section{QuickDASH questionnaire}

The QuickDASH is a validated and widely used questionnaire used to measure arm and shoulder function. It consists of 11 items graded on a 5-point Likert scale. QuickDASH scores indicate the severity of a disability ranging from 0 (no disability) to 100 (severe disability). We recorded QuickDASH scores from each patient at three time points: preoperative, postoperative 6 months, and postoperative 12 months.

\section{Statistical Analysis}

To determine the differences between preoperative and postoperative results, Wilcoxon signed rank tests were performed. Differences between donor site and contralateral control side were determined by Mann-Whitney test. A value of $P<.05$ was considered significant. Statistical analysis was performed with SPSS version 26 (IBM Corp., Armonk, NY, USA).

\section{Results}

A total of 17 patients were initially identified for inclusion in the study. Of these, 4 patients were excluded for not meeting the selection criteria. Therefore, 13 patients with a mean age of 39.6 years (range, 17 to 60 years) were included in the study (Table 1). The mean duration of paralysis was 140.5 months (range, 24 to 660 months). Trauma, brain tumor, and Bell's palsy were the most common etiology of facial palsy, followed by parotid tumor, vestibular schwannoma, and chronic otitis media. Mean follow-up period was 32.2 months (range, 24 to 78 months). Regarding operation-related variables, the mean dimension of harvested latissimus dorsi muscle was $49.8 \mathrm{~cm}^{2}$. The mean length of interfascicular nerve splitting was $7.0 \mathrm{~cm}$ (range, 6.0 to 8.0 $\mathrm{cm}$ ), and the mean length of the harvested descending branch of the TDN was $11.4 \mathrm{~cm}$ (range, 9.0 to 13.0 $\mathrm{cm})$.

All flaps survived without specific complications. Only one patient underwent emergent exploration due to signs of venous thrombosis. However, it was not venous thrombosis but simple swelling of the cheek. Postoperative results of facial reanimation were fair. The average postoperative grade was 3.77, which was statistically different from the preoperative grade. (3.77 \pm 0.73 and 1 , respectively, $P=.001)$. Postoperatively, the lip excursion of the paralyzed side improved from $27.59 \pm 5.31 \mathrm{~mm}$ to $33.39 \pm 4.87 \mathrm{~mm}(P<0.001)$. The difference on the contralateral side decreased significantly from $16.63 \pm 8.71 \mathrm{~mm}$ to $7.54 \pm 4.60(P<0.001)$ (Table 3).

The EMG and NCS grades were evaluated by the rehabilitation department 12 months postoperatively. The donor site grades averaged $0.42 \pm 0.51$. In contrast, the contralateral side averaged $0.08 \pm 0.28$. Only one patient's contralateral side was graded as 1 . There was not a statistically significant difference between donor site and contralateral side $(P=.073)$ (Table 3$)$. The patient graded as 1 postoperatively is shown in Supplementary Content 4.

QuickDASH scores showed different results over time. The average preoperative QuickDASH score was $1.57 \pm 2.34$. At postoperative 6 months, QuickDASH scores averaged $8.74 \pm 4.62$, which were significantly different from the preoperative score $(P=.001)$. At postoperative 12 months, the average QuickDASH score was $2.62 \pm 3.19$, which was an improvement from the postoperative 6 -month average, but not significantly different from the preoperative score $(P=.059)$ (Table 3 and Figure 3 ).

\section{Discussion}

To date, various attempts have been made to reduce donor site functional morbidity and its importance has been emphasized. In the case of free flap surgery, which does not include motor nerves, the development of muscle-sparing flap and perforator flap has dramatically reduced donor site morbidity. The deep inferior 
epigastric perforator flap showed fewer hernia rates than the transverse rectus myocutaneous flap ${ }^{9,10}$ and the muscle-sparing latissimus dorsi flap and thoracodorsal artery perforator flap resulted in better shoulder flap than conventional latissimus dorsi flap function. ${ }^{11,12}$ Donor site morbidity due to sacrifice of sensory nerves has also been reduced through various efforts. For example, in the case of a reverse sural flap, many surgeons tried to prevent sensory deficit by not involving the sural nerve. ${ }^{13,14}$ Recently, an anatomical study has led to a reduction in donor site morbidity using nerve splitting similar to this study. ${ }^{15}$ This study shows a clinical application for this technique for facial reanimation.

One of the important factors for facial reanimation is that nerves are included in the muscle flap. The muscle used as a flap will always induce donor site morbidity due to the sacrifice of the motor nerve, despite a part of the muscle remaining (i.e. deneurotization). One of the most frequently used flaps is the gracilis flap, which has relatively low donor site morbidity and is easy to harvest. ${ }^{16,17}$ However, the biggest disadvantage of the gracilis flap is that it is impossible to reach the contralateral normal side and perform neurorrhaphy without an additional nerve graft because the length of the obturator nerve is short. This leads to another site of morbidity. Latissimus dorsi muscle has a long TDN; therefore, an additional nerve graft is not needed. From estimated clinical results, it could be that the remnant denervated muscle retains part of its function but the exact mechanism of function is not clear. ${ }^{4}$ Instead, we focused on better possible procedure to minimize donor site morbidity.

Currently, the dual-innervation technique is widely performed, and many reports show fair results.,18-21 Faster and more powerful re-innervation through the masseteric nerve as well as spontaneous smile through the contralateral facial nerve can be achieved with this technique. As described above, additional donor site morbidity is inevitable for this, for example, due to sural nerve harvest or transverse branch of the TDN harvest. Also, additional nerve coaptation is needed for dual innervation. Some reports used end-toside coaptation using sural nerve graft for dual innervation. ${ }^{18-20}$ This could induce damage to the motor nerves. Otherwise, using the transverse branch of the TDN needs additional course because its nerve input must go through the descending branch of the TDN. Our technique utilizes the distal stump of the TDN; therefore, additional nerve harvest and nerve coaptation are not necessary. The length of the distal stump of the TDN can be controlled without disturbance; therefore, we can minimize its length and achieve quicker re-innervation.

Interfascicular nerve splitting is not technically difficult, taking approximately 15 minutes. Nevertheless, it should be performed meticulously under microscopy or high-magnified surgical loupes because a small cut could shorten the length of the TDN. Dissecting the distal stump of the TDN also takes approximately 15 minutes. With this procedure, we could achieve similar segmental latissimus dorsi harvest while preserving the transverse branch of the TDN. According to Ding et al., ${ }^{22}$ the QuickDASH score and EMG amplitude at 3 months postoperatively showed no significant difference compared with the preoperative amplitude after segmental latissimus dorsi muscle flap harvest. In this study, the transverse branch of the TDN was preserved. Also, our results showed greater shoulder function improvement compared to other studies regarding donor site morbidity of muscle-sparing latissimus dorsi flap or TDAP flap. The mean QuickDASH scores have ranged from 7.2 within 3 months postoperatively, to 6.42 within 6 months, and 8.2 after 12 months. ${ }^{12,23,24}$ Although it is difficult to compare due to different conditions, our current results reflect a faster recovery time compared to previous studies. Our patients showed the same level of recovery within 12 months compared to at least 3 years of recovery in other studies.

One of our concerns was masseteric nerve input via distal stump of the TDN. Direction of re-innervation is retrograde. Although previous experimental studies have shown that reversing the nerve graft polarity of cable grafts did not affect nerve regeneration electrophysiologically or histologically, ${ }^{25}$ the distal stump of the TDN is different from cable grafts. As expected, the distal stump of the TDN achieved reverse polarity according to signal inputs, confirming previous results. ${ }^{4,21}$ We also observed an insufficient number of axons in the distal stump of the TDN to transfer masseteric nerve input because its diameter was smaller than the TDN descending branch. However, its diameter was comparable with the sural nerve and our clinical results showed sufficient contraction during mastication. [See Video, Supplemental Content 5] 
There are several limitations in this study. First, this was a retrospective chart review and bias could exist. Second, there was a relatively small number of cases; therefore, it does not have statistically powerful results. Third, there was no control group where the whole thoracodorsal nerve was removed. To address this, we compared the donor and contralateral side results. We used the distal stump of the TDN descending branch and reverse innervation to show clinically fair results, but further experimental studies are needed to clarify the mechanism of innervation changes. It is also difficult to clinically evaluate the dual innervation. The border of a spontaneous smile and excursion from mastication are not evident as time goes on. Developing an objective method to evaluate this is a priority for future advancement. Further large-scale, prospective clinical studies and basic research are required.

\section{Conclusions}

The present study showed that interfascicular nerve splitting could minimize donor site morbidity. Our results suggest that the split nerve functions normally as a donor nerve. In addition, using the distal stump of the TDN has made dual innervation possible without any sacrifice of further site morbidity. Our novel method could be a valuable surgical option for facial reanimation to minimize donor site morbidity.

Conflicts of interest: None

\section{Patient Consent}

Patients provided written consent for the use of patients' photographs and videos.

\section{References}

1. Harii K, Asato H, Yoshimura K, Sugawara Y, Nakatsuka T, Ueda K. One-stage transfer of the latissimus dorsi muscle for reanimation of a paralyzed face: a new alternative. Plast Reconstr Surg.1998;102:941-951.

2. Lee KT, Mun GH. A systematic review of functional donor-site morbidity after latissimus dorsi muscle transfer. Plast Reconstr Surg. 2014;134:303-314.

3. Okazaki M, Mutsumi O, Kentaro T, et al. One-stage dual latissimus dorsi muscle flap transfer with a pair of vascular anastomoses and double nerve suturing for long-standing facial paralysis. J Plast Reconstr Aesthet Surg. 2015;68:e113-119.

4. Lee KT, Lee YJ, Kim A, Mun GH. Evaluation of donor morbidity following single-stage latissimus dorsi neuromuscular transfer for facial reanimation. Plast Reconstr Surg. 2019;143:152e-164e.

5. Kwon ST, Chang H, Oh M. Anatomic basis of interfascicular nerve splitting of innervated partial latissimus dorsi muscle flap. J Plast Reconstr Aesthet Surg. 2011;64:e109-114.

6. Cheng A, Audolfsson T, Rodriguez-Lorenzo A, Wong C, Rozen S. A reliable anatomic approach for identification of the masseteric nerve.J Plast Reconstr Aesthet Surg. 2013;66:1438-1440.

7. Bray D, Henstrom DK, Cheney ML, Hadlock TA. Assessing outcomes in facial reanimation: Evaluation and validation of the SMILE system for measuring lip excursion during smiling. Arch Facial Plastic Surg.2010;12:352-354.

8. Guarin DL, Dusseldorp J, Hadlock TA, Jowett N. A machine learning approach for automated facial measurements in facial palsy. JAMA Facial Plast Surg. 2018;20:335-333.

9. Mennie JC, Mohanna PN, O’Donoghue JM, Rainsbury R, Cromwell DA. Donor-site hernia repair in abdominal flap breast reconstruction: A population-based cohort study of 7929 patients. Plast Reconstr Surg. 2015;136:1-9.

10. Jeong W, Lee S, Kim J. Meta-analysis of flap perfusion and donor site complications for breast reconstruction using pedicled versus free TRAM and DIEP flaps. Breast. 2018;38:45-51. 
11. Kim H, Wiraatmadja ES, Lim SY, et al. Comparison of morbidity of donor site following pedicled musclesparing latissimus dorsi flap versus extended latissimus dorsi flap breast reconstruction. J Plast Reconstr Aesthet Surg. 2013;66:640-646.

12. Saint-Cyr M, Nagarkar P, Schaverien M, Dauwe P, Wong C, Rohrich RJ. The pedicled descending branch muscle-sparing latissimus dorsi flap for breast reconstruction. Plast Reconstr Surg. 2009;123:13-24.

13. Motamed S, Yavari M, Mofrad HRH, Rafiee R, Shahraki FN. Distally based sural artery flap without sural nerve. Acta medica Iranica.2010;48:127-129.

14. Aydin OE, Tan O, Kuduban SD, Barin EZ. Nerve sparing-distally based sural flap. Microsurgery. 2011;31:276-280.

15. Kim H, Hu J, Chang H, Kim BJ. Sural Nerve Splitting in Reverse Sural Artery Perforator Flap. Plast Reconstr Surg. 2017;140:1024-1032.

16. Craggs B, Vanmierlo B, Zeltzer A, Buyl R, Haentjens P, Hamdi M. Donor-site morbidity following harvest of the transverse myocutaneous gracilis flap for breast reconstruction. Plast Reconstr Surg.2014;134:682e$691 \mathrm{e}$.

17. Fricke A, Rassner M, Kiefer J, Bannasch H, Stark G, Eisenhardt S. Donor-site morbidity of free muscle and perforator flaps: comparison of the gracilis muscle flap and the anterolateral thigh flap. $J$ Reconstr Microsurg. 2017;33:526-532.

18. Biglioli F, Colombo V, Tarabbia F, et al. Double innervation in free-flap surgery for long- standing facial paralysis. J Plast Reconstr Aesthet Surg. 2012;65:1343-1349.

19. Cardenas-Mejia A, Covarrubias-Ramirez JV, Bello-Margolis A, Rozen S. Double innervated free functional muscle transfer for facial reanimation. J Plast Surg Hand Surg. 2014;49:183-188.

20. Sforza C, Frigerio A, Mapelli A, et al. Double-powered free gracilis muscle transfer for smile reanimation: A longitudinal optoelectronic study. J Plast Reconstr Aesthet Surg. 2015;68:930-939.

21. Watanabe Y, Akizuki T, Ozawa T, Yoshimura K, Agawa K, Ota T. Dual innervation method using onestage reconstruction with free latissimus dorsi muscle transfer for re-animation of established facial paralysis: simultaneous reinnervation of the ipsilateral masseter motor nerve and the contralateral facial nerve to improve the quality of smile and emotional facial expressions. J Plast Reconstr Aesthet Surg.2009;62:15891597 .

22. Ding Y, Cao D, Huang X, Xie J, Li H. Segmental latissimus dorsi free flap attempting to preserve function at the donor site: Anatomical and clinical experiences. J Reconstr Microsurg. 2017;33:268-274.

23. Brackley PTH, Mishra A, Sigaroudina M, Iqbal A. Modified muscle sparing latissimus dorsi with implant for total breast reconstruction - extending the boundaries. J Plast Reconstr Aesthet Surg.2010;63:1495-1502.

24. Izadi D, Paget JTEH, Haj-Basheer M, Khan UM. Fasciocutaneous flaps of the subscapular artery axis to reconstruct large extremity defects.J Plast Reconstr Aesthet Surg. 2012;65:1357-1362.

25. Nakatsuka H, Takamatsu K, Koshimune M, Imai Y, Enomoto M, Yamano Y. Experimental study of polarity in reversing cable nerve grafts. J Reconstr Microsurg. 2002;18:509-515.

\section{Table legends}

Table 1. Patient demographics

Table 2. Electromyography and nerve conduction study criteria for estimation of donor site morbidity

Table 3. Results of estimated donor site morbidity and postoperative results

Figure legends 
Figure 1. Schematic illustration of surgical method using interfascicular nerve splitting and dual-innervation technique. (Left) The flap included thoracodorsal pedicle, spilt thoracodorsal nerve (TDN) and descending branch of the TDN, muscle and distal stump of descending branch of the TDN. (Center) Interfascicular nerve splitting divided transverse branch and descending branch. (Right) Split TDN-descending branch was coapted with buccal branch of the normal side. Distal stump of descending branch of the TDN was coapted with the masseteric motor nerve.

Figure 2. Intraoperative photography that shows interfascicular nerve splitting. (Left) Note the transverse branch, descending branch, and branching point visualized before nerve splitting (Center) After nerve splitting, the transverse branch and descending branch are separated. Distal stump of the thoracodorsal nerve (TDN) is dissected. (Right) Magnified image of two separated nerves, which are linked to the brachial plexus. (yellow arrowhead, transverse branch of the TDN; green arrowhead, descending branch of the TDN)

Figure 3. Quick-Disabilities of Arm, Shoulder, and Hand questionnaire score. Preoperative score and postoperative 6-month score showed significant differences.

\section{Supplemental Contents Legends}

Supplemental Content 1.

Video 1. Stimulation of the transverse branch of the thoracodorsal nerve after splitting shows remnant latissimus dorsi muscle contraction. It demonstrates intact innervation after interfascicular nerve splitting.

\section{Supplemental Content 2.}

Table 1. Evaluation criteria from Harii et. al. (From Harii K, Asato H, Yoshimura K, Sugawara Y, Nakatsuka T, Ueda K. One-stage transfer of the latissimus dorsi muscle for reanimation of a paralyzed face: a new alternative. Plast Reconstr Surg 1998;102;941-51.)

Supplemental Content 3 .

Figure 1. Example of evaluating the lip excursion scale using Emotrics software

Supplemental Content 4.

Figure 2. Example of nerve conduction study and electromyography. Donor site was on the left side. This patient was graded 1 (very mild).

\section{Supplemental Content 5.}

Video 2. A representative case, which demonstrates fair results from dual innervation.

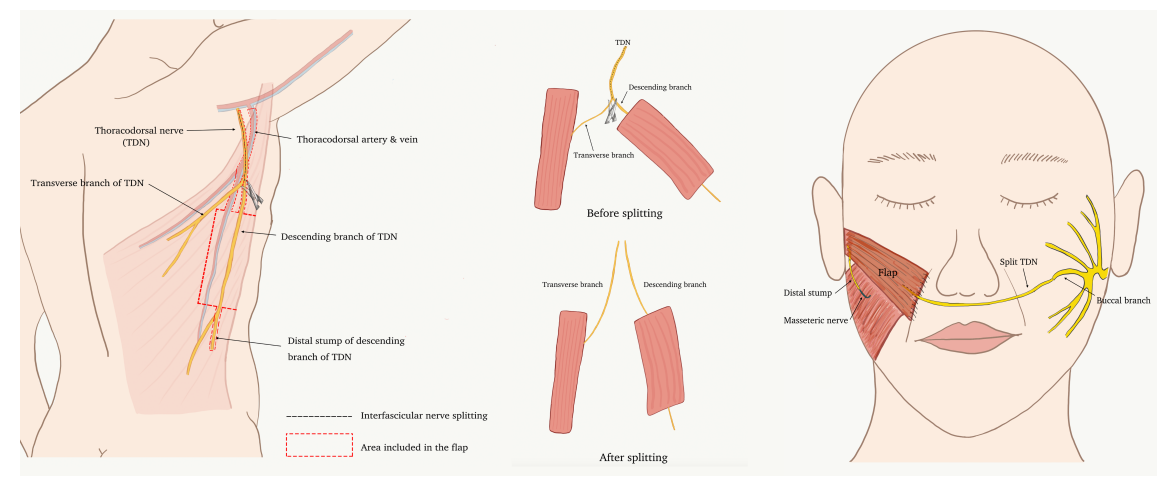



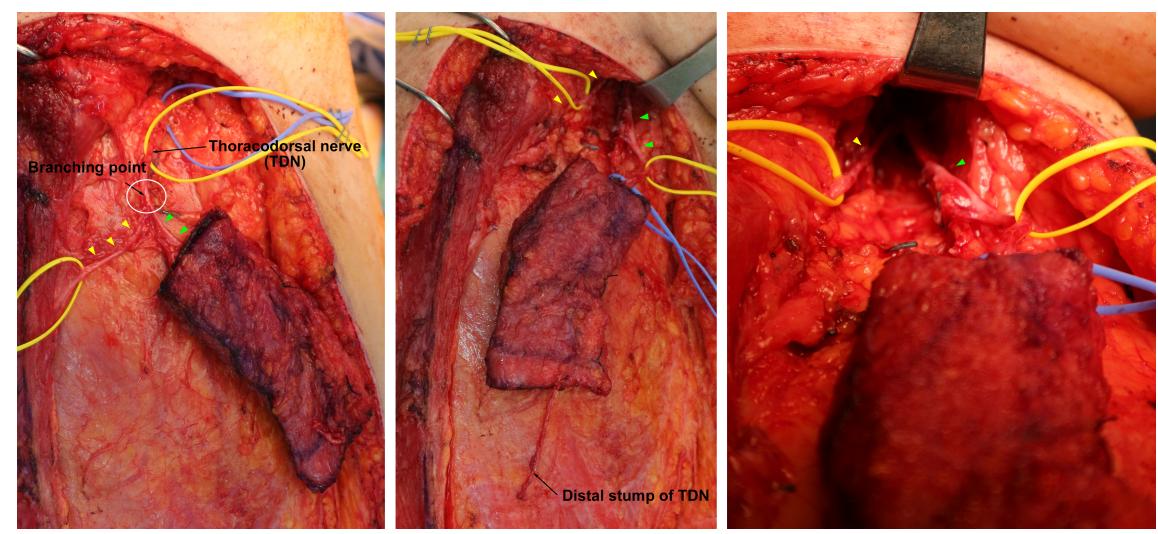

\section{Quick DASH Score Change with Time}

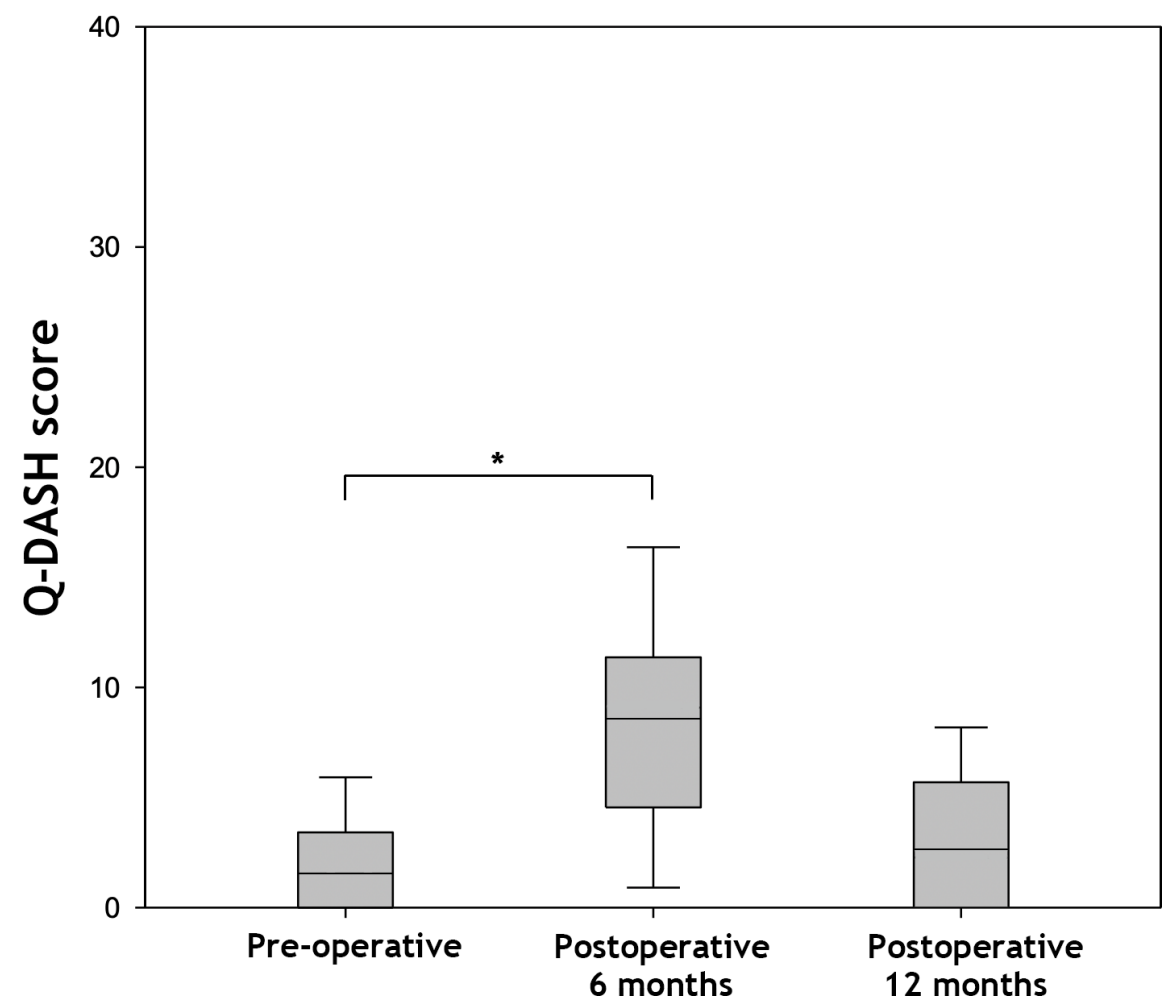

\section{Hosted file}

Table 1.docx available at https://authorea.com/users/334274/articles/460289-minimizingdonor-site-morbidity-using-the-interfascicular-nerve-splitting-technique-in-singlestage-latissimus-neuromuscular-transfer-for-facial-reanimation-a-retrospective-study

Hosted file

Table_2.docx available at https://authorea.com/users/334274/articles/460289-minimizingdonor-site-morbidity-using-the-interfascicular-nerve-splitting-technique-in-singlestage-latissimus-neuromuscular-transfer-for-facial-reanimation-a-retrospective-study 


\section{Hosted file}

Table_3.docx available at https://authorea.com/users/334274/articles/460289-minimizingdonor-site-morbidity-using-the-interfascicular-nerve-splitting-technique-in-singlestage-latissimus-neuromuscular-transfer-for-facial-reanimation-a-retrospective-study 